\title{
Erratum: Robust Extraction of Tomographic Information via Randomized Benchmarking [Phys. Rev. X 4, 011050 (2014)]
}

\author{
Shelby Kimmel \\ Marcus P. da Silva, Colm A. Ryan, Blake R. Johnson, and Thomas Ohki \\ (Received 6 April 2016; published 3 May 2016) \\ Subject Areas: Quantum Physics, Quantum Information
}

DOI: 10.1103/PhysRevX.6.029902

We found errors in the proof of Lemma C.1. However, the statement of Lemma C.1 is still correct, and we give a simpler and correct proof of Lemma C.1 here:

Proof.-Ruskai et al. [1] prove that $\breve{\mathcal{E}}$ is completely positive (CP) if and only if

$$
\begin{gathered}
\left(\lambda_{1}+\lambda_{2}\right)^{2} \leq\left(1+\lambda_{3}\right)^{2}-\tau_{3}^{2}-\left(\tau_{1}^{2}+\tau_{2}^{2}\right)\left(\frac{1+\lambda_{3} \pm \tau_{3}}{1-\lambda_{3} \pm \tau_{3}}\right), \\
\left(\lambda_{1}-\lambda_{2}\right)^{2} \leq\left(1-\lambda_{3}\right)^{2}-\tau_{3}^{2}-\left(\tau_{1}^{2}+\tau_{2}^{2}\right)\left(\frac{1-\lambda_{3} \pm \tau_{3}}{1+\lambda_{3} \pm \tau_{3}}\right), \\
\left(1-\left(\lambda_{1}^{2}+\lambda_{2}^{2}+\lambda_{3}^{2}\right)-\left(\tau_{1}^{2}+\tau_{2}^{2}+\tau_{3}^{2}\right)\right)^{2} \geq 4\left(\lambda_{1}^{2}\left(\tau_{1}^{2}+\lambda_{2}^{2}\right)+\lambda_{2}^{2}\left(\tau_{2}^{2}+\lambda_{3}^{2}\right)+\lambda_{3}^{2}\left(\tau_{3}^{2}+\lambda_{1}^{2}\right)-2 \lambda_{1} \lambda_{2} \lambda_{3}\right),
\end{gathered}
$$

where in Eqs. (1) and (2), if $\left|\lambda_{3}\right|+\left|\tau_{3}\right|=1$, then $\tau_{1}$ and $\tau_{2}$ must be 0 for the map to be CP.

Notice that if these conditions are satisfied for a completely positive and trace preserving (CPTP) map $\breve{\mathcal{E}}$, then they are also satisfied for the map $\breve{\mathcal{E}}^{-}$, which is the same as $\breve{\mathcal{E}}$, except with $\tau_{1} \rightarrow-\tau_{1}, \tau_{2} \rightarrow-\tau_{2}, \tau_{3} \rightarrow-\tau_{3}$. Hence $\breve{\mathcal{E}}^{-}$must also be CPTP. Now the convex combination of CPTP maps is also CPTP, so $1 / 2\left(\breve{\mathcal{E}}^{-}+\breve{\mathcal{E}}\right)=\breve{\mathcal{E}}^{\prime}$ is CPTP.

[1] M. B. Ruskai, S. Szarek, and E. Werner, An Analysis of Completely-Positive Trace-Preserving Maps on $M_{2}$, Linear Algebra Appl. 347, 159 (2002).

Published by the American Physical Society under the terms of the Creative Commons Attribution 3.0 License. Further distribution of this work must maintain attribution to the author(s) and the published articles title, journal citation, and DOI. 